\title{
DESIGN OF A NOVEL MICROSTRIP MIMO ANTENNA SYSTEM WITH IMPROVED ISOLATION
}

\author{
Y.V.S.S.SatyaMitra ${ }^{1, S k . K a l i s h a}{ }^{1}$, G.Sindhu ${ }^{1}$, P.Raghavendra Kumar ${ }^{1}$, P.Satish ${ }^{1}$ \\ andDr. K. JagadeeshBabu ${ }^{2}$ \\ ${ }^{1}$ Students of Dept. of ECE, SACET, Chirala-523187, AP, India \\ ${ }^{2}$ Professor \& Head of the Dept. ECE, SACET, Chirala-523187, AP, India
}

\begin{abstract}
A novel compact two element MIMO (Multiple Input Multiple Output) antenna system for WLAN applications with improved isolation characteristics is presented in this paper. The proposed antenna resonates at $5.4 \mathrm{GHz}$ frequency with good return loss characteristics. A $2 \times 2$ MIMO antenna system is developed using the proposed antenna giving good isolation of $-31 \mathrm{~dB}$ between the two antennas. The surface current distribution and radiation pattern of the proposed MIMO system are also presented. The developed antenna system can be used in modern wireless systems employing MIMO technology.
\end{abstract}

\section{Keywords}

microstrip antenna,MIMO, Isolation, surface current, radiation pattern

\section{INTRODUCTION}

Numerous Microstrip patch antennas turned enormously popular in mobile and radio wireless communication due to their convenience in investigation, low cost to fabricate as they can be printed directly on to the circuit board and their interesting radiation features. In this modernistic generation, the demand for the antennas that can accommodate more than one communication standards in a single compact system is broadly increased like tri-band [1] or multiband antennas.

Based on miniaturization and multiband operations numerous antennas were developed by the researchers to meet the demands of present wireless communication. Along with the physical constraints, comfort in designing and outlook are few other important aspects to be considered while designing the MIMO system. In general the spacing between the elements in an array cannot be extended beyond a certain level which restrict in achieving the desired spectral efficiencies. When working on small Personal Digital Assistants (PDA) the major problem faced by the MIMO system [2] engineers is mutual coupling, which mainly arises due to the smaller spacing between the antenna elements. The primary plan while designing the MIMO systems is to reduce mutual coupling parameter that describes the correlation between the received signals in highly diversified environments, which degrades the performance of the communication system [3].

In MIMO systems, the spacing between the elements should be as minimum as possible as they have major applications in portable devices like laptops, mobile terminals, and WLAN access points [4], where size of the device is to be maintained as small as possible. The surface current flowing through the ground surface is one of the main sources of mutual coupling. Several DOI: $10.5121 /$ jant.2016.2203 
techniques like Electromagnetic band gap structure [5], defected ground structure [6], decoupling techniques, etc. are studied in the literature to reduce these surface currents flowing on the ground surface. However, these methods are costlier to implement and are complex.

Microstrip antennas turned very common in the 1970s mainly for space borne engineering. Now they are used for concerning business and government applications. The microstrip antenna offers conformable, low-profile to planar and non-planar surfaces, cheap and simple to fabricate using advanced printed-circuit technology and very variable in terms of frequency, resonant, polarization, impedance and radiation patterns. The popular designs in the literature are E shaped patch antenna [7], $\mathrm{H}$ shaped patch antenna [8], U slotted patch antennas [9], etc., giving wide bandwidth and good isolation when employed in MIMO systems.

In the present work, a novel compact two element MIMO antenna system is proposed with improved isolation. The designed antenna resonates at $5.4 \mathrm{GHz}$. The designed antenna as a two element array reduces mutual coupling between the antenna elements because of its structural design and obtained a mutual coupling of $-31 \mathrm{~dB}$. Section 2 presents the proposed antenna geometry and Section 3 describes the design of $2 \times 2$ MIMO antenna system.

\section{Antenna Geometry}

The Figure 1 shows the design and dimensions of the proposed antenna. The substrate selected for the design of the proposed antenna is Rogers RT Duroid $5880(\mathrm{tm})$ with thickness $1.6 \mathrm{~mm}$ and with low permittivity $\left(\varepsilon_{\mathrm{r}}=2.2\right)$. The dimensions of the substrate are taken as $30 \times 50 \times 1.6 \mathrm{~mm}^{3}$. The co-axial probe is used to feed the whole system at the position $(\mathrm{X} 0, \mathrm{Y} 0)=(17.0,3.0)$ as coaxial feeding is simpler to implement.

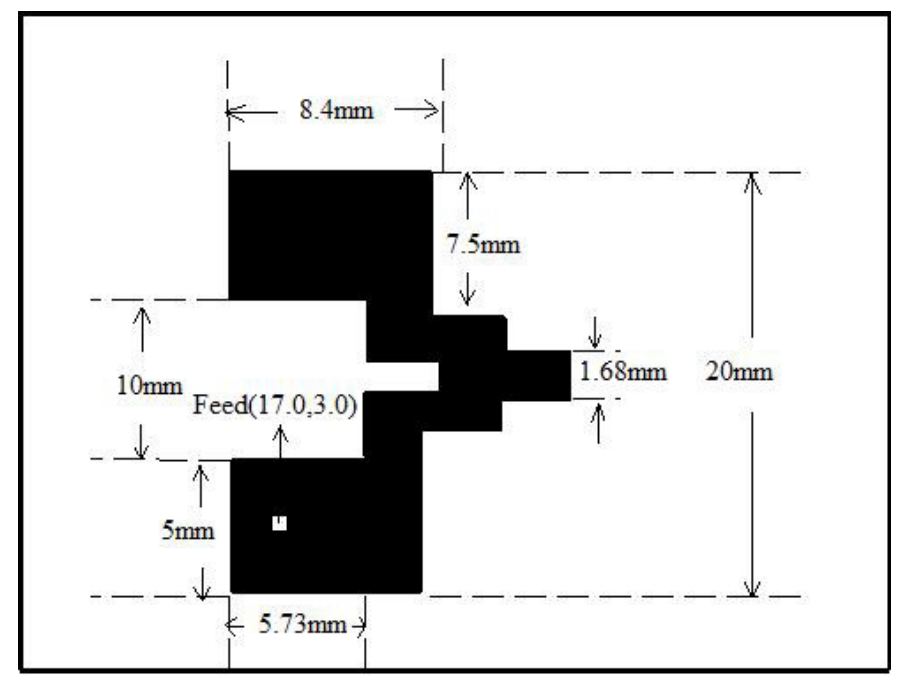

Figure 1. Proposed microstrip patch antenna 
The mutual coupling is more pronounced, when the adjacent antennas distance is less than $\lambda / 4$. For compact devices such as in handheld mobile phones or PDAs and USB dongles, MIMO antennas are very closely placed giving high isolation. These high coupling levels will degrade the efficiency and capacity of the MIMO system. Based on the antenna structure and its radiation and feeding mechanisms several methods have been suggested in literature to eliminate or minimize such coupling effect. The reflection coefficient of the proposed antenna is shown in Figure 2, resonating at $5.4 \mathrm{GHz}$ frequency, making the antenna system suitable for many Wireless applications including IEEE 802.11a.

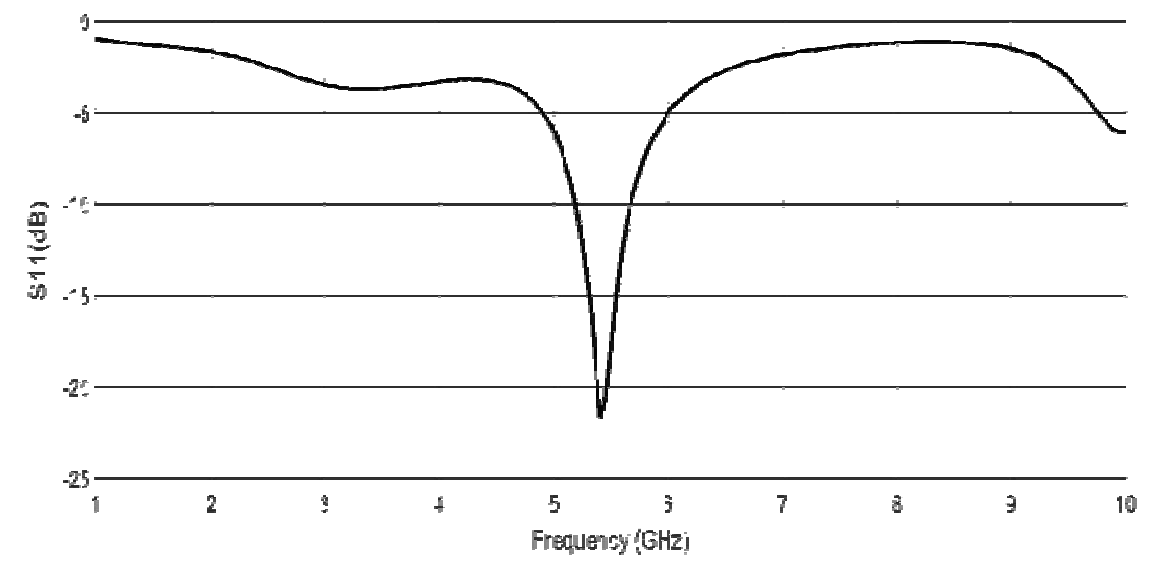

Figure 2. Reflection coefficient of the proposed antenna

\section{DESIGN OF $2 \times 2$ MIMO SYSTEM}

Mutual Coupling is the main parameter that affects the performance of the MIMO system. The design issues are more complicated when multiple antennas are involved at closer spacing compared to a SISO (Single Input Single Output) system. By properly choosing the shape of the antenna, the reduction in mutual coupling can be achieved without increasing the distance between the elements and also without using any complex structures like EBG or DGS or parasitic elements.

Using the proposed microstrip antenna, a $2 \times 2$ MIMO system is developed as shown in Figure 3. The separation between the elements is taken as $10 \mathrm{~mm}$ in the proposed MIMO system, while maintaining the same dimensions of the basic antenna. The surface current distribution of the proposed MIMO antenna array system is shown in Fig. 4. The Figure 5 shows the s-parameters of the proposed $2 \times 2 \mathrm{MIMO}$ antenna system. The system resonates at $5.4 \mathrm{GHz}$ and the obtained mutual coupling between the antenna elements is very small and is $-31 \mathrm{~dB}$. The main reason for achieving these high isolation characteristics is due to the structural arrangement of the proposed microstrip antenna for the proposed MIMO system. The return loss, resonant frequencies and isolation of the proposed $2 \times 2$ MIMO system can be seen in Figure 5. The radiation pattern of the proposed MIMO system is shown in Fig.6. 


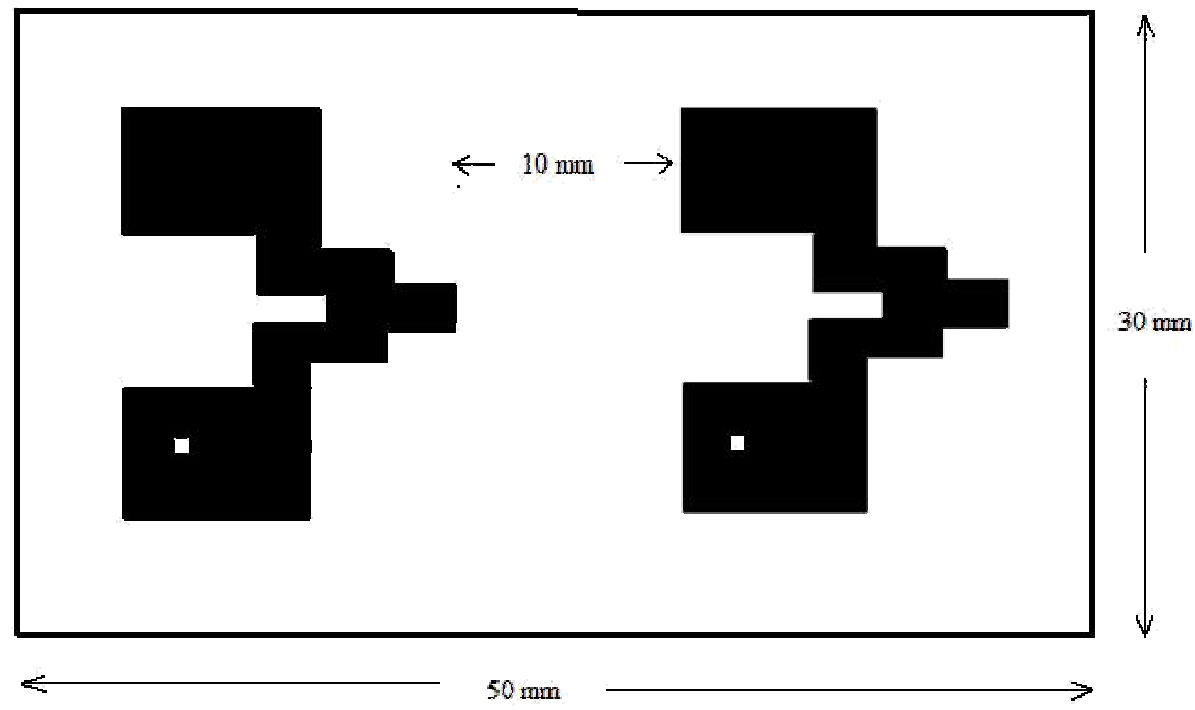

Figure 3. A two element MIMO system using proposed microstrip antenna
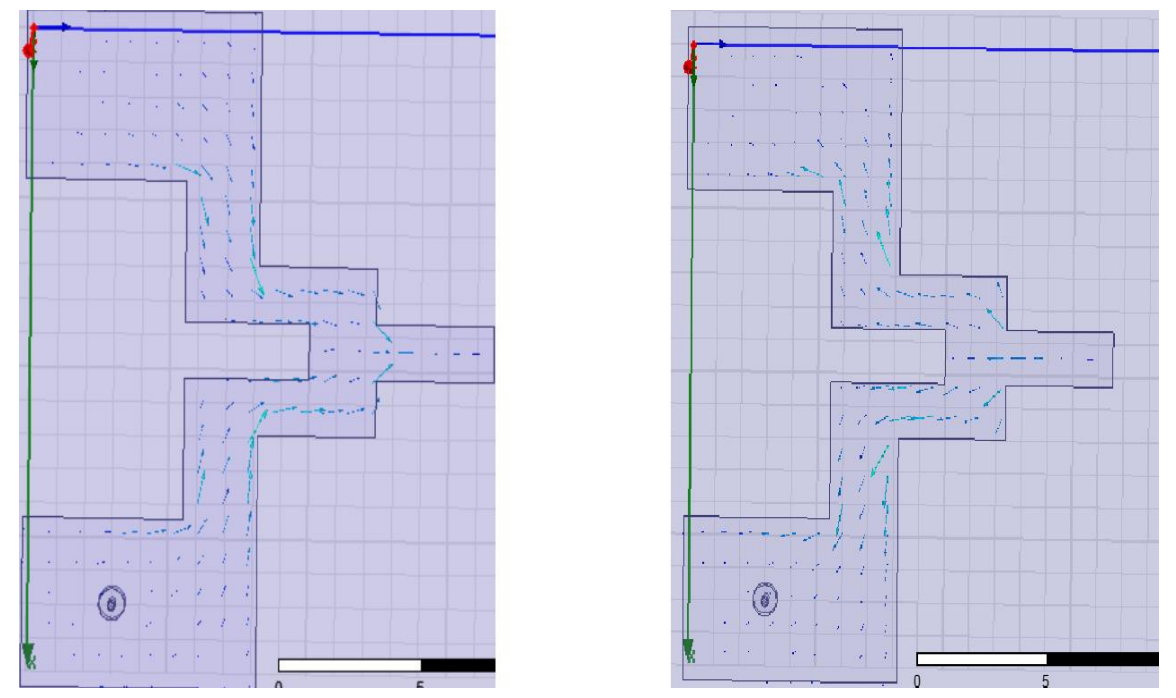

Figure 4. Surface Current Distribution of the proposed antenna system 
International Journal of Antennas (JANT) Vol.2, No.2, April 2016

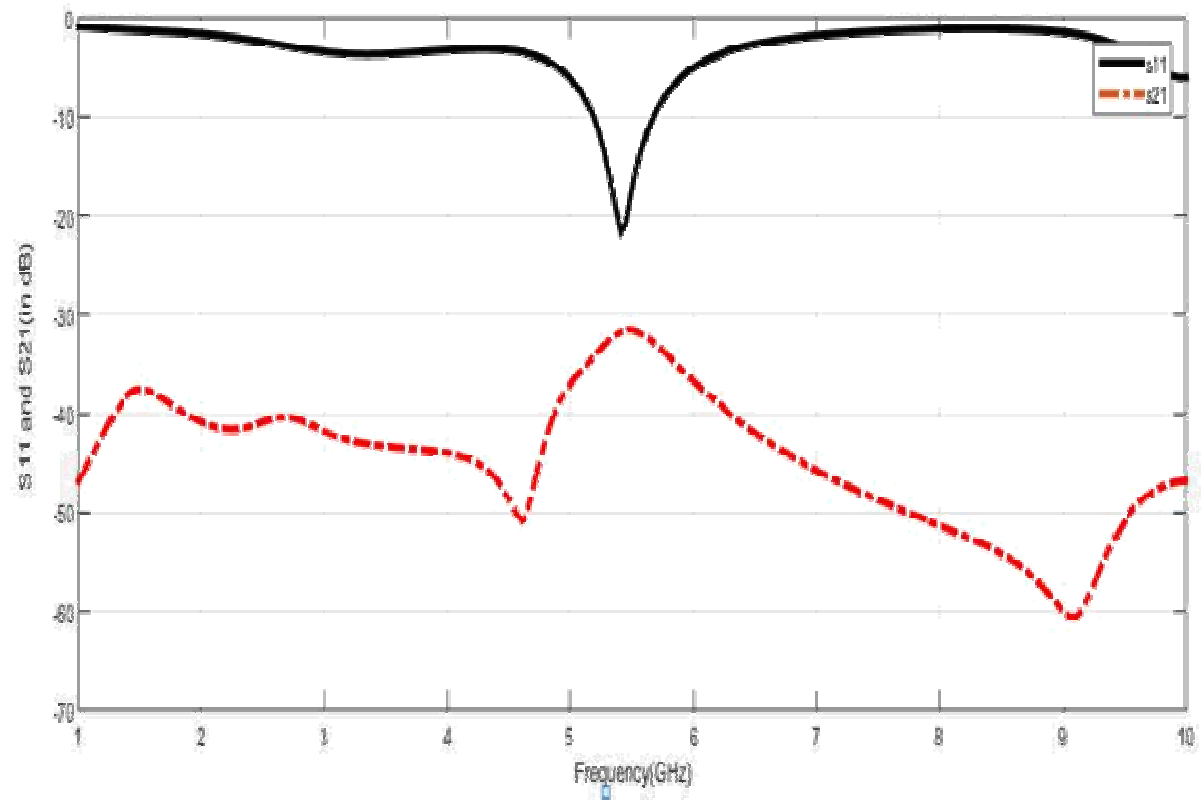

Figure 5. S parameters of proposed microstrip $2 \times 2$ MIMO antennas System
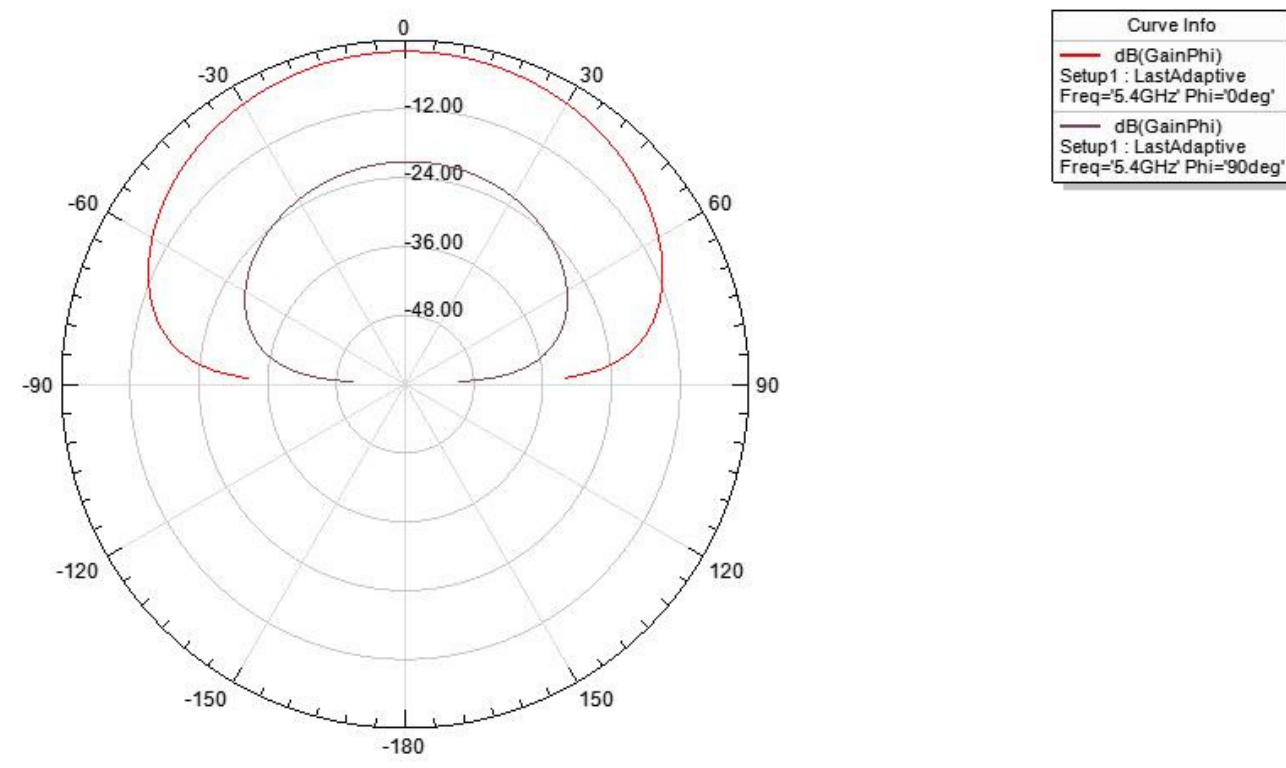

Figure 6. Radiation Pattern of the Proposed Antenna 


\section{CONCLuSiON}

MIMO antenna systems will also be used for next generation wireless terminals, i.e. 5G and beyond. For every new wireless generation the data rates and channel capacities are growing. Hence, a very large leap is expected by the year 2020 where data rates in the ranges of tens of gigabits per second are expected from mobile terminals and other compact devices. In this paper, a novel compact two element MIMO array is developed resonating at $5.4 \mathrm{GHz}$ with a reduced mutual coupling of $-31 \mathrm{~dB}$. These characteristics are well suitable for MIMO applications, using which high data rates can be obtained. We can further improve the channel capacity by employing more number of antennas in the MIMO system.

\section{References}

[1] K. J. Babu, K. S. R. Krishna and L. P. Reddy, "A triband swastika shaped patch antenna with reduced mutual coupling for wireless MIMO systems", Journal of Electronics (China), SPRINGER, vol. 28, pp. 483-487, November 2011.

[2] K. J. Babu, K. S. R. Krishna and L. P. Reddy, "A review on the design of MIMO antennas for upcoming 4G communications", International Journal of Applied Engineering Research, Dindigul, vol. 1, no. 4, 2011.

[3] A. A. Abouda and S. G. Hgagman, "Effect of mutual coupling capacity of MIMO wireless channels in high SNR scenario", Progress In Electromagnetics Research, PIER 65, pp. 27-40, 2006.

[4] M. A. Jensen and J. W. Wallace, "A review of antennas and propagation for MIMO wireless communications", IEEE Trans. Antennas Propagation, vol. 52, pp. 2810-2824, November 2004.

[5] F. Caminita, S. Costanzo, G. DiMassa, G. Guarnieri, S. Maci, G. Mauriello and I. Venneri,

"Reduction of patch antenna coupling by using a compact EBG formed by shorted strips with interlocked branch stubs", IEEE Antennas and Wireless Propagation Letters, vol. 8, no.1, pp. 811- 814, 2009.

[6] F. Fan and Zehongyan, "Compact band pass filter with spurious pass band suppression using defected ground structure", Microwave and optical Technology Letters, vol. 52, no. 1, pp. 17-20, 2009.

[7] P. N. Kumar, K. M. Vijay, T. Srinivas and K. J. Babu, "A Modified Back to Back E-Shaped Patch Antenna for 4G MIMO Communications", International Journal of Engineering and Technology, vol.

2, no. 3, March 2012.

[8] S. C. Gao, L. W. Li, M. S. Leong and T. S. Yeo, "Analysis of an H-shaped patch antenna by using the FDTD Method", Progress in Electromagnetics Research, vol. 34, no. 1, pp. 165-187, (2001).

[9] R. Chair, C. Mak, K. Lee, K. Luk and A. A. Kishk, "Miniature wideband half U-slot and half E-shaped patch antennas", IEEE Transactions on Antennas and Propagation, vol. 53, no. 8, pp. 2645- 2652, 2005. 


\section{Authors}

Y.V.S.S.SatyaMitracompleted the B.Tech degree in Electronics and CommunicationEngineering in 2016 from the JNTU University, Kakinada. His areas of interest include Microstrip Antennas, MIMO Antennas, and Wireless communications.

Sk.Kalishacompleted the B.Tech degree in Electronics and Communication Engineering in2016 from the JNTU University, Kakinada. His areas of interest include Microstrip Antennas, MIMO Antennas, and Wireless communications.

G.Sindhucompleted the B.Tech degree in Electronics and Communication Engineering in 2016from the JNTU University, Kakinada. Her areas of interest includeMicrostrip Antennas, MIMO Antennas, and Wireless communications.

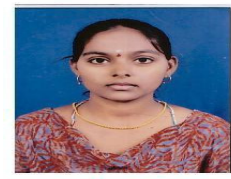

P.Raghavendra Kumar completed the B.Tech degree in Electronics and CommunicationEngineering in 2016 from the JNTU University, Kakinada. His areas of interest include MicrostripAntennas, MIMO Antennas, and Wireless communications.

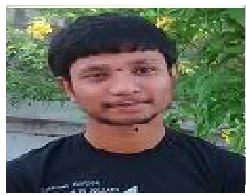

P.Satishcompleted the B.Tech degree in Electronics and Communication Engineering in 2016from the JNTU University, Kakinada. His areas of interest include Microstrip Antennas, MIMOAntennas, and Wireless communications.

KamiliJagadeeshBabuis working as Professor and Head in E.C.E Department at St. Ann'sCollege of Engineering \& Technology, Chirala, India. He obtained his Ph.D. degree from JNTUH, Hyderabad, India in 2013. His areas of interest include design of Microstrip and Dielectric Resonator Antennas for MIMO applications and UWB antennas for wireless applications. He published more than 20 research papers in referred journals. He is currently a reviewer for many reputed journals

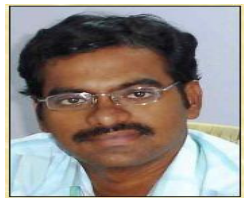
such as ACES, International Journal of Electronics Letters (Taylor \& Francis), IET Microwaves, Antennas and Propagation, IET Electronics Letters etc. He is a Member of IEEE Antennas and Propagation Society and ISTE. 